\title{
Interfacial ferroelectricity by van der Waals sliding
}

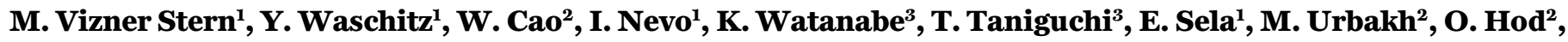 \\ M. Ben Shalom ${ }^{1 *}$ \\ ${ }^{1}$ School of Physics and Astronomy, Tel Aviv University, Israel. ${ }^{2}$ Department of Physical Chemistry, School of Chemistry, The Raymond and Beverly Sackler Faculty of Exact \\ Sciences and The Sackler Center for Computational Molecular and Materials Science, Tel Aviv University, Tel Aviv 6997801, Israel. ${ }^{3}$ National Institute for Material Science, \\ Tsukuba, Japan. \\ *Corresponding author. E-mail: moshebs@tauex.tau.ac.il
}

Despite their partial ionic nature, many layered diatomic crystals avoid internal electric polarization by forming a centrosymmetric lattice at their optimal van-der-Waals stacking. Here, we report a stable ferroelectric order emerging at the interface between two naturally-grown flakes of hexagonal-boronnitride, which are stacked together in a metastable non-centrosymmetric parallel orientation. We observe alternating domains of inverted normal polarization, caused by a lateral shift of one lattice site between the domains. Reversible polarization switching coupled to lateral sliding is achieved by scanning a biased tip above the surface. Our calculations trace the origin of the phenomenon to a subtle interplay between charge redistribution and ionic displacement, and provide intuitive insights to explore the interfacial polarization and its unique "slidetronics" switching mechanism.

The ability to locally switch a confined electrical polarization is a key functionality in modern technologies, where storing and retrieving a large volume of information is vital (1). The need to reduce the dimensions of individually polarized domains, from the $\sim 100 \mathrm{~nm}^{2}$ scale $(2,3)$ toward the atomic scale is rising (4). The main challenges involve long-range dipole interactions, which tend to couple the individual domain polarization orientations (5). Likewise, surface effects and external strains that are difficult to control become dominant once the surface-to-volume ratio increases (6). To overcome these challenges, one can consider layered materials, such as hexagonal-boron-nitride $(h$-BN) and transition-metal-dichalcogenides (TMD), where the bulk volume can be reduced to the ultimate atomic-thickness limit whereas the crystalline surface remains intact (7). However, it is rare to find a spontaneous net electric polarization in $2 \mathrm{D}$ that is sufficiently large to read and write under ambient conditions $(8-10)$. Furthermore, in naturally-grown $h$-BN and TMD crystals, polarization is eliminated by the formation of a centrosymmetric van der Waals (vdW) structure that is lower in energy than other meta-stable stacking configurations. Here, we break this symmetry by controlling the twist-angle between two $h$-BN flakes, and find an array of permanent and switchable polarization domains at their interface. The polarization is oriented normal to the plane, and its amplitude is in good agreement with previous first-principle predictions for a two-layered system (11) and with our detailed multi-layer calculations.

To identify which stacking modes can carry polarization, we present in Fig. 1A six different high symmetry configurations of bilayer $h$-BN. The stacking configurations are divided into two groups termed "parallel" and "anti-parallel" twist orientations (12); within each group, a relative lateral shift by one interatomic distance switches the stacking configuration in a cyclic manner. Typically, the crystal grows in the optimal anti-parallel (AA') configuration with full overlap between nitrogen (boron) atoms of one layer and boron (nitrogen) atoms of the adjacent layer (13). In the parallel twist-orientation, however, the fully eclipsed configuration (AA) is unstable since it forces pairs of bulky nitrogen atoms atop each other resulting in increased steric repulsion (14). Instead, a lateral interlayer shift occurs to a metastable $\mathrm{AB}$ stacking with only half of the atoms overlapping, whereas the other half are aligned with the empty centers of the hexagons in the adjacent layer $(15,16)$. Note that the $\mathrm{AB}$ and $\mathrm{BA}$ stacking form equivalent lattice structures (only flipped), and that all depicted anti-parallel configurations (AA', $\mathrm{AB1}^{\prime}, \mathrm{AB} 2$ ') are symmetric under spatial inversion.

To explore these different configurations, we artificially stamped two exfoliated $h$-BN flakes on top of each other, each consisting of a few AA' stacked layers, with a minute twistangle between the otherwise parallel interfacial layers (17). The small twist imposes interlayer translations that evolve continuously and form a Moiré pattern owing to the underlying crystal periodicity (Fig. 1B). In this rigid lattice picture, the three nearly commensurate stacking configurations (AB, BA, AA) appear at adjacent positions in space. Notably, this picture breaks for a sufficiently small twist angle as a result of structural relaxation processes as shown by our molecular dynamics calculation based on dedicated interlayer potentials (Fig. 1C, $(17,18)$ ). Instead, the system divides into large domains of reconstructed commensurate $\mathrm{AB}$ and $\mathrm{BA}$ stackings separated by sharp incommensurate domain walls that accommodate the global twist (see fig. S3, A and B) (19-22). Notably, near the center of the extended commensurate 
domains, perfectly aligned configurations are obtained with no interlayer twist. In the experiments, we also introduce a topographic step at the interface between the flakes. A step thickness of an odd number of layers guarantees anti-parallel stacking ( $\mathrm{AA}^{\prime}, \mathrm{AB1}^{\prime}$ or $\mathrm{AB2}$ ') on one side, and parallel stacking (AA, AB or BA) on its other side (Fig. 1D). Thus, one can compare all possible configurations at adjacent locations in space.

To measure variations in the electrical potential, $\mathrm{V}_{\mathrm{KP}}$, at surface regions of different stacking configurations, we place the $h$-BN sandwich on a conducting substrate $\left(\mathrm{Si}_{\mathrm{SiO}}\right.$, graphite, or gold), and scan an atomic force microscope (AFM) operated in a Kelvin probe mode (KPFM), see Fig. 2A and (17). The potential map above the various stacking configurations is shown in Fig. 2B. We find clear domains (black and white) of constant $V_{\mathrm{KP}}$, extending over areas of several $\mu \mathrm{m}^{2}$, which are separated by narrow domain-walls. Dark gray areas of average potential are observed above: (i) positions where only one $h$-BN flake exists (outside the dashed yellow line); (ii) above two flakes but beyond the topographic step marked by dashed red lines in Fig. 2B (and topography map fig. S2) as expected; and (iii) beyond topographic folds that can further modify the interlayer twist angle (dashed green lines). These findings confirm that white and black domains correspond to $\mathrm{AB}$ and $\mathrm{BA}$ interfacial stacking that host a permanent out-ofplane electric polarization. Such polarization is not observed at the other side of the step, where centrosymmetric AA', AB1' AB2' configurations are obtained, or at the AA configuration expected at domain-wall crossings (see blue dots in Fig. 1C). Sufficiently far from the domain-walls (toward the center of each domain) a constant potential is observed with a clear difference $\Delta V_{K P}$ between the $\mathrm{AB}$ and $\mathrm{BA}$ domains as shown in Fig. 2C. We note that KPFM measurement nullifying the tip response at the electric bias frequency give an underestimated potential difference of $\Delta V_{K P} \sim 100 \mathrm{mV}$ due to averaging contributions from the tip's cantilever (17). More quantitative measurements obtained via sideband tip excitations yield $\Delta V_{K P}$ values ranging between 210 and $230 \mathrm{mV}$ for both closed-loop scans and local open-loop measurements (fig. S1). Similar values are measured for several samples with different substrate identity, various thicknesses of the top $h$ $\mathrm{BN}$ flake (for flakes thicker than $1 \mathrm{~nm}$ ), and using different AFM tips. These findings confirm that $\Delta V_{K P}$ is an independent measure of the intrinsic polarization of the system that, in turn, is confined within a few interfacial layers.

Although our force field calculations for slightly twisted bilayer $h$-BN show a uniform triangular lattice of alternating $\mathrm{AB}$ and $\mathrm{BA}$ stacked domains (Fig. 1C), in the experiment we observe large variations in their lateral dimensions and shape. This indicates minute deviations in the local twist, which are unavoidable in the case of small twist angles (1922). Specifically, the $\sim 1 \mu \mathrm{m}^{2}$ domains in the left part of Fig.
2B correspond to a global twist of less than $0.01^{\circ}$ (23). We also note that any external perturbation to the structure, caused either by transferring it to a polymer, heating, or directly pressing it with the AFM tip, usually resulted in a further increase in domain size. In a few cases, high-temperature annealing resulted in a global reorientation to a single domain flake, many micrometers in dimensions. This behavior confirms the metastable nature of the $\mathrm{AB}$ / BA stacking mode, as well as the possible superlubric nature of the interface (24, 25 ). On the other extreme, much smaller domains are observed in the top right-hand section of Fig. 2B. The smallest triangle edge we could identify over many similar flakes was $60 \mathrm{~nm}$ in length, which corresponds to a twist-angle of $0.24^{\circ}$. We therefore conclude that, below this angle, atomic reconstruction to create untwisted domains is energetically favored. Naturally, this constitutes a lower bound on the maximal angle for domain formation as smaller domains below our experimental resolution may form at larger twist angles.

To trace the microscopic origin of the measured polarization we performed a set of density functional theory (DFT) calculations on finite bilayer and quad-layer $h$-BN flakes. For the finite bilayer calculations, we constructed two model systems, where hydrogen passivated $h$-BN flakes of either $1 \mathrm{~nm}^{2}$ or $3 \mathrm{~nm}^{2}$ surface area are stacked in the $\mathrm{AB}$ stacking mode (fig. S4). The calculated polarizations per unit area, $P_{z} / A$, of these systems were $0.55,0.45$ Debye $/ \mathrm{nm}^{2}$, respectively (see black triangles in Fig. 2D), pointing perpendicular to the interface only (see table S1). Because edge effects may influence the results of such finite system calculations (17), fig. S4), we performed complementary laterally periodic system calculations at various thicknesses. The detailed methods used for these calculations are discussed in (17). For the AB stacked periodic bilayer, we find a polarization of $P_{z} / A=0.33$ Debye $/ \mathrm{nm}^{2}$, changing by only $10 \%$ when including additional two and eight $\mathrm{AA}^{\prime}$ stacked layers above and below the $\mathrm{AB}$ stacked interface (see Fig. 2D and fig. S7). Adding more $\mathrm{AB}$-stacked layers, however, reveals a linear increase of the calculated polarization with the number of added interfaces (see fig. S9). The latter is a highly appealing control-mechanism to engineer the magnitude of the polarization in future 2D systems, independent of external effects such as surface-chemistry and local strains. Lateral shifts of the periodic bilayer system show a gradual evolution of the polarization when shifting between the $\mathrm{AA}, \mathrm{AB}$, and $\mathrm{BA}$ stacking modes, from zero to +0.33 and -0.33 Debye $/ \mathrm{nm}^{2}$, respectively (Fig. 2E). This is crucial when considering the complex response at domain-walls, where lattice deformations induce additional flexo (26) and piezo (27) responses. In fig. S8 we present also the charge density redistribution in the periodic 
bilayer system owing to interlayer coupling. The corresponding interlayer potential difference at the experimental configuration is calculated to be $\frac{1}{2} \Delta V_{K P}=120 \mathrm{mV}$ (see fig. S7), in excellent agreement with the side-band measurements (red star in Fig. 2D). Similar results were reported in recent computational studies (11). We note, however, that to obtain quantitative agreement with the experimental measurements, the results should be carefully converged with respect to the various calculation parameters (figs. S5 and S7). By further applying an electric field of $0.1 \mathrm{~V} / \mathrm{nm}$ normal to the plane, our calculations find a minute piezo-electric deformation of $0.1 \mathrm{pm}$, and a $5 \%$ variation in the polarization magnitude (fig. S10).

It is instructive to further translate the measured potential difference into intra-layer displacements in a simplistic point-like charges model (see $\Delta d$ in Fig. 1D), where each atom is allowed to displace from its layer's basal plane in the vertical direction. Note that using the lattice site density of $n=37 \mathrm{~nm}^{-2}$, and the on-site charge value, $q \sim e / 2$, for singlelayered $h$-BN $(28,29)$, our measured $\Delta V_{K P}$ gives out-of-plane atomic displacement of the order of $\Delta d=\Delta V_{K P} \varepsilon_{0} / 4 n q \sim 1 \times 10^{-3} \AA \quad\left(\varepsilon_{0}\right.$ is vacuum permittivity), which is much smaller than the intralayer (1.44 $\AA$ ) and interlayer ( $3.30 \AA)$ spacings. This implies that the polarization is determined by a delicate competition between the various interlayer interaction components and charge redistribution. Intuitively, we expect the vdW attraction to vertically compress the non-overlapping interfacial sites (diagonal dashed ellipse in Fig. 1D) closer together than the overlapping sites (vertical dashed ellipse in Fig. 1D), which are more prone to Pauli repulsion. This direction of motion, for example, reduces the average interlayer separation and favors Bernal (AB like) stacking in graphite over the AA configuration (30). In $h$-BN, however, the partial ionic nature of the two lattice sites $(12,31,32)$ makes the fully eclipsed $\mathrm{AA}^{\prime}$ stacking more stable (13). Hence, imposing a polar AB interface, as in our case, may favor overlapping sites of opposite charges to come closer together than the non-overlapping pairs and the polarization to point in the opposite direction.

To quantify these arguments, we present a reduced classical bilayer model that captures the intricate balance between Pauli, vdW, and Coulomb interatomic interactions at different stacking modes. In our model (17), the interfacial energy $E=\frac{1}{2} \sum_{i, j}\left[4 \varepsilon\left(\left(\frac{\sigma}{r_{i j}}\right)^{12}-\left(\frac{\sigma}{r_{i j}}\right)^{6}\right)+\frac{q_{i} q_{j}}{r_{i j}}\right]$, includes a Lennard-

Jones (LJ) potential characterized by the cohesive energy, $\varepsilon$, and the interlayer spacing scale $\sigma$, and Coulomb interactions between the dimensionless partial atomic charges on the boron and nitrogen sites $q= \pm q_{i} / e$. Although neglecting any charge transfer processes between the layers that are explicitly taken into account in our DFT calculations, this model captures both the magnitude and orientation of the polarization by adjusting the ratio between $\varepsilon$ and Coulomb scales $\propto \varepsilon / q^{2}$ (see fig. S11). We note that our detailed DFT calculations indicate that in bilayer $h$-BN the net polarization is oriented as marked by the arrows in Fig. 1D.

The permanent polarization observed in separated domains, whose dimensions can be tuned by the twist-angle, each exhibiting a distinct and stable potential, may be useful in applications. To that end, however, one should identify additional ways to control the local orientation beyond the twisting mechanism. Note that reversible switching between $\mathrm{AB}$ and $\mathrm{BA}$ configurations, accompanied by polarization inversion, can be achieved via relative lateral translation by one atomic spacing (1.44 $\AA$ ) as illustrated in Fig. 1A. Similar sliding between different stacking configurations was recently demonstrated in multilayered graphene. It was shown that both mechanical (33) or electric perturbations (34) can push domain-walls, practically modifying the local stacking. In the present $h$-BN interface, however, the polar switching calls for a preferred up or down orientation that can be predetermined by the user. To obtain such a spatially resolved control we scanned a biased tip above an individual domain to induce a local electric field normal to the interface. The polarization images before and after the biased scans are presented in Fig. 3. We observe a redistribution of domain-walls to orient the local polarization with the electric field under the biased tip. For example, after scanning a negatively biased tip above the region marked by the blue square, we observed a large white domain thanks to the motion of the walls marked by dashed red (green) line before (after) the scan. A successive scan by a positively biased tip resulted in practically complete domain polarization flipping. Hence, by applying negative or positive bias to the tip it is possible to determine the polarization orientation of the underlying domain. Similar switching behavior was attained above different domains within the same interface and for several measured structures (fig. S12). We note that domain-wall motion is observed for electric field values exceeding $\sim 0.3 \mathrm{~V} / \mathrm{nm}$, and when operating the biased scan in a pin-point mode (17).

Our results therefore demonstrate that the broken symmetry at the interface of parallel-stacked $h$-BN flakes gives rise to an out-of-plane two-dimensional polarization confined within a few interfacial layers, that can be locally detected and controlled. While the $h$-BN system, with only two different light atoms, offers a convenient experimental and computational testbed and allows for intuitive interpretations, first-principle analysis (11) predicts similar phenomena to occur in other, more complex bi-atomic vdW crystals, such as 
various TMDs $(35,36)$. Notably, the origin of the polarization and the sliding inversion mechanism presented herein are fundamentally different from the minute deformations of tightly-bonded atoms in common non-centrosymmetric 3D bulk crystals. The "slidetronics" switching involves lateral motion by a full lattice spacing in a weakly-coupled interface under ambient conditions. The associated sliding order-parameter reveals vortices patterns around the AA points (see Fig. 1C and Fig. 2B) with topological aspects resembling the hexagonal manganite system (37). Unlike the 3D manganites, the present 2D structure allow relaxation processes through the delamination and formation of bubbles, or the annealing of walls at the open edges (see fig. S12). In the present study, however, we focus on the physics away for the domain wall and toward the domain center, where no twist, moire pattern, or strain are considered.. The sensitivity of the system to the delicate interplay between van der Waals attraction, Pauli repulsion, Coulomb interactions, and charge redistribution implies that external stimuli such as pressure, temperature, and/or electric fields may be used to control the polarization, thus offering many opportunities for future research.

We note that (38) reports similar findings. During the review process of this manuscript, similar experimental findings were also reported in (39), and a ferroelectric response in aligned bilayer graphene was reported in (40).

\section{REFERENCES AND NOTES}

1. M. Lines, A. Glass, Principles and applications of ferroelectrics and related materials (2001;

https: / www.google.com/books?hl=en\&lr=\&id=p6ruJH8C84kC\&oi=fnd\&pg=PA $1 \& \mathrm{dq}=$ Principles+and+Applications+of+Ferroelectrics+and+Related+Materials + (Oxford+University,+Press, +1977)\&ots=3F7179vH7T\&sig=nefFtl1fv8lkap4UyS1S 30rvUro).

2. N. Setter, D. Damjanovic, L. Eng, G. Fox, S. Gevorgian, S. Hong, A. Kingon, H. Kohlstedt, N. Y. Park, G. B. Stephenson, I. Stolitchnov, A. K. Taganstev, D. V. Taylor, T. Yamada, S. Streiffer, Ferroelectric thin films: Review of materials, properties, and applications. J. Appl. Phys. 100, 051606 (2006). doi:10.1063/1.2336999

3. J. F. Scott, [3D] nano-scale ferroelectric devices for memory applications. Ferroelectrics 314, 207-222 (2005). doi:10.1080/00150190590926490

4. J. F. Scott, Prospects for Ferroelectrics: 2012-2022. ISRN Mater. Sci. 2013, 1-24 (2013). doi:10.1155/2013/187313

5. M. Dawber, K. M. Rabe, J. F. Scott, Physics of thin-film ferroelectric oxides. Rev. Mod. Phys. 77, 1083-1130 (2005). doi:10.1103/RevModPhys.77.1083

6. J. Müller, P. Polakowski, S. Mueller, T. Mikolajick, Ferroelectric Hafnium Oxide Based Materials and Devices: Assessment of Current Status and Future Prospects. ECS J. Solid State Sci. Technol. 4, N30 (2015). doi:10.1149/2.0081505jss

7. Y. Cao, A. Mishchenko, G. L. Yu, E. Khestanova, A. P. Rooney, E. Prestat, A. V. Kretinin, P. Blake, M. B. Shalom, C. Woods, J. Chapman, G. Balakrishnan, I. V. Grigorieva, K. S. Novoselov, B. A. Piot, M. Potemski, K. Watanabe, T. Taniguchi, S. J. Haigh, A. K. Geim, R. V. Gorbachev, Quality Heterostructures from TwoDimensional Crystals Unstable in Air by Their Assembly in Inert Atmosphere. Nano Lett. 15, 4914-4921 (2015). doi:10.1021/acs, nanolett. 5 b00648 Medline

8. D. D. Fong, G. B. Stephenson, S. K. Streiffer, J. A. Eastman, O. Aucielo, P. H. Fuoss, C. Thompson, Ferroelectricity in ultrathin perovskite films. Science 304, 16501653 (2004). doi:10.1126/science.1098252

9. S. S. Cheema, D. Kwon, N. Shanker, R. Dos Reis, S.-L. Hsu, J. Xiao, H. Zhang, R. Wagner, A. Datar, M. R. McCarter, C. R. Serrao, A. K. Yadav, G. Karbasian, C.-H.
Hsu, A. J. Tan, L.-C. Wang, V. Thakare, X. Zhang, A. Mehta, E. Karapetrova, R. V. Chopdekar, P. Shafer, E. Arenholz, C. Hu, R. Proksch, R. Ramesh, J. Ciston, S. Salahuddin, Enhanced ferroelectricity in ultrathin films grown directly on silicon. Nature 580, 478-482 (2020). doi:10.1038/s41586-020-2208-x Medline

10. F. Liu, L. You, K. L. Seyler, X. Li, P. Yu, J. Lin, X. Wang, J. Zhou, H. Wang, H. He, S. T. Pantelides, W. Zhou, P. Sharma, X. Xu, P. M. Ajayan, J. Wang, Z. Liu, Roomtemperature ferroelectricity in CulnP 2 S 6 ultrathin flakes. Nat. Commun. 7, 1-6 (2016).

11. L. Li, M. Wu, Binary Compound Bilayer and Multilayer with Vertical Polarizations: Two-Dimensional Ferroelectrics, Multiferroics, and Nanogenerators. ACS Nano 11 6382-6388 (2017). doi:10.1021/acsnano.7b02756 Medline

12. G. Constantinescu, A. Kuc, T. Heine, Stacking in bulk and bilayer hexagonal boron nitride. Phys. Rev. Lett. 111, 036104 (2013). doi:10.1103/PhysRevLett.111.036104 Medline

13. R. S. Pease, Crystal structure of boron nitride. Nature 165, 722-723 (1950). doi:10.1038/165722b0 Medline

14. S. Zhou, J. Han, S. Dai, J. Sun, D. J. Srolovitz, Van der Waals bilayer energetics: Generalized stacking-fault energy of graphene, boron nitride, and graphene/boron nitride bilayers. Phys. Rev. B Condens. Matter Mater. Phys. 92, 155438 (2015). doi:10.1103/PhysRevB.92.155438

15. S. M. Gilbert, T. Pham, M. Dogan, S. Oh, B. Shevitski, G. Schumm, S. Liu, P. Ercius, S. Aloni, M. L. Cohen, A. Zettl. Alternative stacking sequences in hexagonal boron nitride. 2D Mater. 6, 021006 (2019). doi:10.1088/2053-1583/ab0e24

16. J. H. Warner, M. H. Rümmeli, A. Bachmatiuk, B. Büchner, Atomic resolution imaging and topography of boron nitride sheets produced by chemical exfoliation. ACS Nano 4, 1299-1304 (2010). doi:10.1021/nn901648g Medline

17. Materials, methods, and additional information are available as supplementary materials.

18. T. Maaravi, I. Leven, I. Azuri, L. Kronik, O. Hod, Interlayer Potential for Homogeneous Graphene and Hexagonal Boron Nitride Systems: Reparametrization for Many-Body Dispersion Effects. J. Phys. Chem. C 121, 22826-22835 (2017). doi:10.1021/acs.jpcc.7b07091

19. J. S. Alden, A. W. Tsen, P. Y. Huang, R. Hovden, L. Brown, J. Park, D. A. Muller, P. L. McEuen, Strain solitons and topological defects in bilayer graphene. Proc. Natl. Acad. Sci. U.S.A. 110, 11256-11260 (2013). doi:10.1073/pnas.1309394110 Medline

20. M. R. Rosenberger, H. J. Chuang, M. Phillips, V. P. Oleshko, K. M. McCreary, S. V. Sivaram, C. S. Hellberg, B. T. Jonker, Twist Angle-Dependent Atomic Reconstruction and Moiré Patterns in Transition Metal Dichalcogenide Heterostructures. ACS Nano 14, 4550-4558 (2020). doi:10.1021/acsnano.0c00088 Medline

21. A. Weston, Y. Zou, V. Enaldiev, A. Summerfield, N. Clark, V. Zólyomi, A. Graham, C. Yelgel, S. Magorrian, M. Zhou, J. Zultak, D. Hopkinson, A. Barinov, T. H. Bointon, A. Kretinin, N. R. Wilson, P. H. Beton, V. I. Fal'ko, S. J. Haigh, R. Gorbachev, Atomic reconstruction in twisted bilayers of transition metal dichalcogenides. Nat. Nanotechnol. 15, 592-597 (2020). doi:10.1038/s41565-020-0682-9 Medline

22. H. Yoo, R. Engelke, S. Carr, S. Fang, K. Zhang, P. Cazeaux, S. H. Sung, R. Hovden, A. W. Tsen, T. Taniguchi, K. Watanabe, G.-C. Yi, M. Kim, M. Luskin, E. B. Tadmor, E. Kaxiras, P. Kim, Atomic and electronic reconstruction at the van der Waals interface in twisted bilayer graphene. Nat. Mater. 18, 448-453 (2019). doi:10.1038/s41563-019-0346-z Medline

23. T. A. Green, J. Weigle, Theorie du moire. Helv. Phys. Acta 21, 217 (1948),

24. C. R. Woods, L. Britnell, A. Eckmann, R. S. Ma, J. C. Lu, H. M. Guo, X. Lin, G. L. Yu, Y. Cao, R. V. Gorbachev, A. V. Kretinin, J. Park, L. A. Ponomarenko, M. I. Katsnelson, Y. N. Gornostyrev, K. Watanabe, T. Taniguchi, C. Casiraghi, H.-J. Gao, A. K. Geim, K. S. Novoselov, Commensurate-incommensurate transition in graphene on hexagonal boron nitride. Nat. Phys. 10, 451-456 (2014). doi:10.1038/nphys2954

25. O. Hod, E. Meyer, Q. Zheng, M. Urbakh, Structural superlubricity and ultralow friction across the length scales. Nature 563, 485-492 (2018) doi:10.1038/s41586-018-0704-z Medline

26. L. J. McGilly, A. Kerelsky, N. R. Finney, K. Shapovalov, E. M. Shih, A. Ghiotto, Y. Zeng, S. L. Moore, W. Wu, Y. Bai, K. Watanabe, T. Taniguchi, M. Stengel, L. Zhou, J. Hone, X. Zhu, D. N. Basov, C. Dean, C. E. Dreyer, A. N. Pasupathy, Visualization of moiré superlattices. Nat. Nanotechnol. 15, 580-584 (2020). 
doi:10.1038/s41565-020-0708-3 Medline

27. P. Ares, T. Cea, M. Holwill, Y. B. Wang, R. Roldán, F. Guinea, D. V. Andreeva, L. Fumagalli, K. S. Novoselov, C. R. Woods, Piezoelectricity in Monolayer Hexagonal Boron Nitride. Adv. Mater. 32, e1905504 (2020). doi:10.1002/adma.201905504 Medline

28. W. M. Lomer, K. W. Morton, The electrostatic energy of boron nitride. Proc. Phys. Soc. A 66, 772-773 (1953). doi:10.1088/0370-1298/66/8/117

29. Y.-N. Xu, W. Y. Ching, Calculation of ground-state and optical properties of boron nitrides in the hexagonal, cubic, and wurtzite structures. Phys. Rev. B Condens. Matter 44, 7787-7798 (1991). doi:10.1103/PhysRevB.44.7787 Medline

30. J.-C. Charlier, X. Gonze, J.-P. Michenaud, Graphite Interplanar Bonding: Electronic Delocalization and van der Waals Interaction. Europhys. Lett. 28, 403-408 (1994). doi:10.1209/0295-5075/28/6/005

31. N. Marom, J. Bernstein, J. Garel, A. Tkatchenko, E. Joselevich, L. Kronik, O. Hod, Stacking and registry effects in layered materials: The case of hexagonal boron nitride. Phys. Rev. Lett. 105, 046801 (2010). doi:10.1103/PhysRevLett.105.046801 Medline

32. 0. Hod, Graphite and hexagonal boron-nitride have the same interlayer distance. Why? J. Chem. Theory Comput. 8, 1360-1369 (2012). doi:10.1021/ct200880m Medline

33. L. Jiang, S. Wang, Z. Shi, C. Jin, M. I. B. Utama, S. Zhao, Y. R. Shen, H. J. Gao, G. Zhang, F. Wang, Manipulation of domain-wall solitons in bi- and trilayer graphene. Nat. Nanotechnol. 13, 204-208 (2018). doi:10.1038/s41565-017-0042-6 Medline

34. M. Yankowitz, J. I. J. Wang, A. G. Birdwell, Y. A. Chen, K. Watanabe, T. Taniguchi, P. Jacquod, P. San-Jose, P. Jarillo-Herrero, B. J. LeRoy, Electric field control of soliton motion and stacking in trilayer graphene. Nat. Mater. 13, 786-789 (2014). doi:10.1038/nmat3965 Medline

35. J. Sung, Y. Zhou, G. Scuri, V. Zólyomi, T. I. Andersen, H. Yoo, D. S. Wild, A. Y. Joe, R. J. Gelly, H. Heo, S. J. Magorrian, D. Bérubé, A. M. M. Valdivia, T. Taniguchi, K. Watanabe, M. D. Lukin, P. Kim, V. I. Fal'ko, H. Park, Broken mirror symmetry in excitonic response of reconstructed domains in twisted $\mathrm{MoSe}_{2} / \mathrm{MoSe}_{2}$ bilayers. Nat. Nanotechnol. 15, 750-754 (2020). doi:10.1038/s41565-020-0728-z Medline

36. T. I. Andersen, G. Scuri, A. Sushko, K. de Greve, J. Sung, Y. Zhou, D. S. Wild, R. J. Gelly, H. Heo, K. Watanabe, T. Taniguchi, P. Kim, H. Park, M. D. Lukin, Moiré excitons correlated with superlattice structure in twisted WSe2/WSe2 homobilayers. arXiv (2019) (available at https://arxiv.org/abs/1912.06955).

37. S. Artyukhin, K. T. Delaney, N. A. Spaldin, M. Mostovoy, Landau theory of topological defects in multiferroic hexagonal manganites. Nat. Mater. 13, 42-49 (2014). doi:10.1038/nmat3786 Medline

38. K. Yasuda, X. Wang, K. Watanabe, T. Taniguchi, P. Jarillo-Herrero, Stackingengineered ferroelectricity in bilayer boron nitride. Science 10.1126/science.abd3230 (2021). https://doi.org/10.1126/science.abd3230

39. C. R. Woods, P. Ares, H. Nevison-Andrews, M. J. Holwill, R. Fabregas, F. Guinea, A. K. Geim, K. S. Novoselov, N. R. Walet, L. Fumagalli, Charge-polarized interfacial superlattices in marginally twisted hexagonal boron nitride. Nat. Commun. 12, 347 (2021). doi:10.1038/s41467-020-20667-2 Medline

40. Z. Zheng, Q. Ma, Z. Bi, S. de la Barrera, M. H. Liu, N. Mao, Y. Zhang, N. Kiper, K. Watanabe, T. Taniguchi, J. Kong, W. A. Tisdale, R. Ashoori, N. Gedik, L. Fu, S. Y. Xu, P. Jarillo-Herrero, Unconventional ferroelectricity in moiré heterostructures. Nature 588, 71-76 (2020). doi:10.1038/s41586-020-2970-9 Medline

41. M. Vizner Stern, Y. Waschitz, W. Cao, I. Nevo, K. Watanabe, T. Taniguchi, E. Sela, M. Urbakh, O. Hod, M. Ben Shalom, Replication Data for: Interfacial Ferroelectricity by van der Waals Sliding, Zenodo (2021). https://doi.org/10.5281/zenodo.4746309.

42. C. R. Dean, A. F. Young, I. Meric, C. Lee, L. Wang, S. Sorgenfrei, K. Watanabe, T. Taniguchi, P. Kim, K. L. Shepard, J. Hone, Boron nitride substrates for high-quality graphene electronics. Nat. Nanotechnol. 5, 722-726 (2010). doi:10.1038/nnano.2010.172 Medline

43. W. Melitz, J. Shen, A. C. Kummel, S. Lee, Kelvin probe force microscopy and its application. Surf. Sci. Rep. 66, 1-27 (2011). doi:10.1016/i.surfrep.2010.10.001

44. R. Borgani, D. Forchheimer, J. Bergqvist, P. A. Thorén, O. Inganäs, D. B. Haviland, Intermodulation electrostatic force microscopy for imaging surface photovoltage. Appl. Phys. Lett. 105, 143113 (2014). doi:10.1063/1.4897966

45. J. Colchero, A. Gil, A. M. Baró, Resolution enhancement and improved data interpretation in electrostatic force microscopy. Phys. Rev. B Condens. Matter
Mater. Phys. 64, 245403 (2001). doi:10.1103/PhysRevB.64.245403

46. C. Sevik, A. Kinaci, J. B. Haskins, T. Çağın, Characterization of thermal transport in low-dimensional boron nitride nanostructures. Phys. Rev. B Condens. Matter Mater. Phys. 84, 085409 (2011). doi:10.1103/PhysRevB.84.085409

47. I. Leven, R. Guerra, A. Vanossi, E. Tosatti, O. Hod, Multiwalled nanotube faceting unravelled. Nat. Nanotechnol. 11, 1082-1086 (2016). doi:10.1038/nnano.2016.151 Medline

48. P. C. Hariharan, J. A. Pople, The influence of polarization functions on molecular orbital hydrogenation energies. Theor. Chim. Acta 28, 213-222 (1973). doi:10.1007/BF00533485

49. M. J. Frisch, G. W. Trucks, H. B. Schlegel, G. E. Scuseria, M. A. Robb, J. R. Cheeseman, G. Scalmani, V. Barone, G. A. Petersson, H. Nakatsuij, X. Li, M. Caricato, A. Marenich, J. Bloino, B. G. Janesko, R. Gomperts, B. Mennucci, H. P. Hratchian, J. V. Ortiz, A. F. Izmaylov, J. L. Sonnenberg, D. Williams-Young, F. Ding, F. Lipparini, F. Egidi, J. Goings, B. Peng, A. Petrone, T. Henderson, D. Ranasinghe, V. G. Zakrzewski, J. Gao, N. Rega, G. Zheng, W. Liang, M. Hada, M. Ehara, K. Toyota, R. Fukuda, J. Hasegawa, M. Ishida, T. Nakajima, Y. Honda, O. Kitao, H. Nakai, T. Vreven, K. Throssell, J. A. Montgomery Jr., J. E. Peralta, F. Ogliaro, M. Bearpark, J. J. Heyd, E. Brothers, K. N. Kudin, V. N. Staroverov, T. Keith, R. Kobayashi, J. Normand, K. Raghavachari, A. Rendell, J. C. Burant, S. S. Iyengar, J. Tomasi, M. Cossi, J. M. Millam, M. Klene, C. Adamo, R. Cammi, J. W. Ochterski, R. L. Martin, K. Morokuma, O. Farkas, J. B. Foresman, D. J. Fox, Gaussian 09, Rev. D.01 (Gaussian Inc., Wallingford, CT, 2016).

50. S. Grimme, J. Antony, S. Ehrlich, H. Krieg, A consistent and accurate ab initio parametrization of density functional dispersion correction (DFT-D) for the 94 elements H-Pu. J. Chem. Phys. 132, 154104 (2010). doi:10.1063/1.3382344 Medline

51. F. Weigend, R. Ahlrichs, Balanced basis sets of split valence, triple zeta valence and quadruple zeta valence quality for $\mathrm{H}$ to $\mathrm{Rn}$ : Design and assessment of accuracy. Phys. Chem. Chem. Phys. 7, 3297-3305 (2005). doi:10.1039/b508541a Medline

52. G. Kresse, J. Furthmüller, Efficient iterative schemes for ab initio total-energy calculations using a plane-wave basis set. Phys. Rev. B Condens. Matter Mater. Phys. 54, 11169-11186 (1996). doi:10.1103/PhysRevB.54.11169 Medline

53. J. P. Perdew, K. Burke, M. Ernzerhof, Generalized gradient approximation made simple. Phys. Rev. Lett. 77, 3865-3868 (1996). doi:10.1103/PhysRevLett.77.3865 Medline

54. G. Kresse, D. Joubert, From ultrasoft pseudopotentials to the projector augmented-wave method. Phys. Rev. B Condens. Matter Mater. Phys. 59, 17581775 (1999). doi:10.1103/PhysRevB.59.1758

55. A. Ambrosetti, A. M. Reilly, R. A. DiStasio Jr., A. Tkatchenko, Long-range correlation energy calculated from coupled atomic response functions. J. Chem. Phys. 140, A508 (2014). doi:10.1063/1.4865104 Medline

56. P. Zhao, C. Xiao, W. Yao, Universal superlattice potential for 2D materials from twisted interface inside h-BN substrate. arXiv (2020) (available at https://arxiv.org/abs/2011.03933).

57. C. Kittel, Introduction to Solid State Physics (Wiley, ed. 8, 2004).

58. P. P. Gillis, Calculating the elastic constants of graphite. Carbon 22, 387-391 (1984). doi:10.1016/0008-6223(84)90010-1

\section{ACKNOWLEDGMENTS}

We thank Yoav Lahini for useful discussions, Andrea Cerreta from Park Systems for AFM support, and Neta Ravid for laboratory support. We further thank Prof. Juan E. Peralta for helpful discussions regarding the Density Functional Theory calculations. Funding: W.C. acknowledges the financial support of the IASH and the Sackler Center for Computational Molecular and Materials Science at Tel Aviv Univeristy. Growth of hBN was supported by the Elemental Strategy Initiative conducted by the MEXT, Japan, Grant Number JPMXP 0112101001 JSPS KAKENHI Grant Number JP20H00354 and the CREST (JPMJCR15F3), JST E.S. acknowledges support from ARO (W911NF-20-1-0013), the Israel Science Foundation grant number 154/19 and US-Israel Binational Science Foundation (Grant No. 2016255). M.U. acknowledges financial support of the Israel Science Foundation, Grant 1141/18 and the ISFNSFC joint Grant 3191/19. O.H. is grateful for the generous financial support of the Israel Science Foundation under Grant 1586/17, the Naomi Foundation for generous financial support via the 2017 Kadar Award, and the Ministry of Science and Technology of Israel under project 
number 3-16244. M.B.S. acknowledge funding by the European Research Council (ERC) under the European Union's Horizon 2020 research and innovation programme (grant agreement No.852925), the Israel Science Foundation Grant No. 1652/18, and the Israel Ministry of Science \& Technology project number 3-15619 (Meta-Materials). O.H. and M.B.S acknowledge the Center for Nanoscience and Nanotechnology of Tel-Aviv University. Author contributions: M.V.S. and Y.W. performed the experiments, supported by I.N. and supervised by M.B.S.; W.C. performed the DFT calculations supervised by M.U. and O.H.; K.W. and T.T grew the hBN crystals; E.S. devised the adhesion model; All authors contributed to the writing of the manuscript. Competing interests: Ramot at Tel Aviv University Ltd. has applied for a patent (US application no. 63/083,947) on some of the technology and materials discussed here, on which M.V.S., Y.W. and M.B.S. are listed as co-inventors. Data and materials availability: All data needed to evaluate the conclusions in the study are present in the main text or the supplementary materials. The data have also been uploaded to Zenodo (41).

\section{SUPPLEMENTARY MATERIALS}

science.sciencemag.org/cgi/content/full/science.abe8177/DC1

Materials and Methods

Figs. S1 to S12

Table S1

References (42-59)

16 September 2020; accepted 10 May 2021

Published online 10 June 2021

10.1126/science.abe8177 


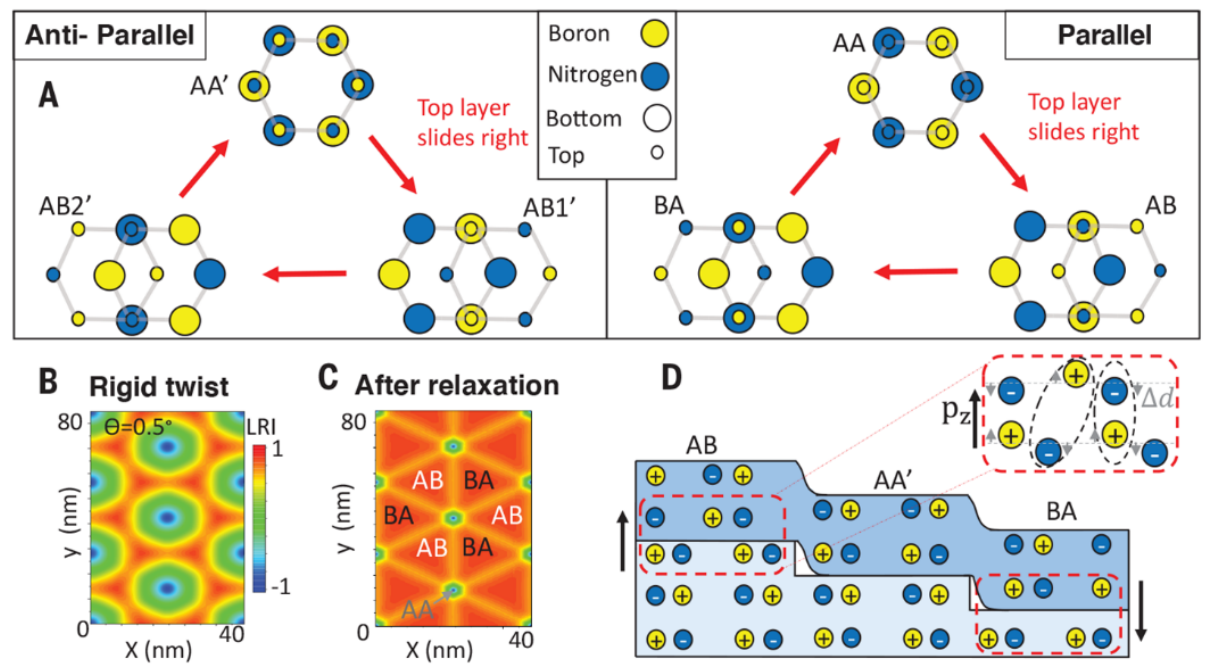

Fig. 1. High symmetry interlayer stacking configurations. (A) Top view illustration of two layers. For clarity, atoms of the top layer are represented by smaller circles. Within each group of parallel / anti-parallel twist orientations, a relative lateral shift by one lattice spacing results in a cyclic switching between three high-symmetry stacking configurations. (B) Calculated local-registry index (LRI) map of the atomic overlaps, see (17), in a rigid structure made of two $h B N$ layers stacked with a twist angle of $0.5^{\circ}$. Blue regions correspond to AA stacking, whereas AB/BA stacking appear in orange $(\mathrm{LRI}=0.86)$. (C) Calculated LRI map after geometry relaxation of the structure presented in $(B)$. Large domains of uniform untwisted $A B / B A$ stacking appear, at the expense of the pre-optimized $A A$ regions. The twist is accumulated in smaller AA-like regions and in the $10 \mathrm{~nm}$ wide incommensurate domain walls (bright lines), see fig. S3 and (17) for further discussion. (D) Cross-sectional illustration of two fewlayered flakes (blue and light blue regions marked the top and bottom flakes, respectively) of naturally grown $h-B N\left(A^{\prime}\right)$, which are stacked with no twist. Plus (minus) signs mark boron (nitrogen) sites. A topographical step of a single layer switches between parallel and anti-parallel stacking orientations at the interface between the two flakes. Vertical charge displacements and the resulting net polarization $\mathrm{P}_{z}$ are marked by arrows. 

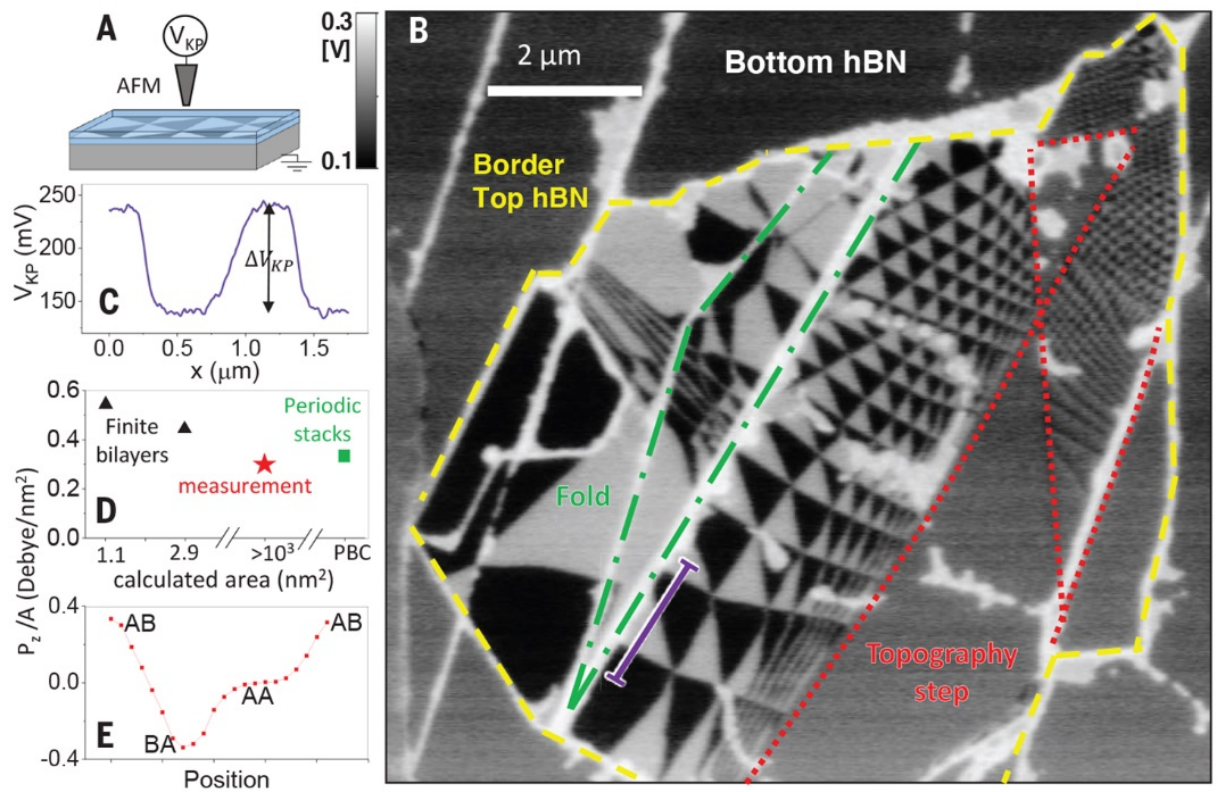

Fig. 2. Direct measurement of interfacial polarization. (A) Illustration of the experimental setup. An atomic force microscope is operated in Kelvinprobe mode to measure the local potential modulation, $V_{K P}$, at the surface of two $3 \mathrm{~nm}$ thick $h$-BN flakes, which are stacked with a very small twist angle. (B) $V_{K P}$ map showing oppositely-polarized domains of AB/BA stacking (black and white), ranging in area between $\sim 0.01$ and $1 \mu \mathrm{m}^{2}$ and separated by sharp domain-walls. (C) Surface potential measured along the purple line marked in (B) by first-harmonic KPFM. (D) DFT calculations of the polarization, $P_{z}$, per unit area obtained for finite $h$-BN bilayer flakes of different lateral dimensions (1.1 and $2.9 \mathrm{~nm}^{2}$, black triangles) and for laterally periodic stacks made of 2-10 layers (marked as PBC, green square). The red star marks the polarization value evaluated from the measured $\Delta V_{K P}$ data. (E) Calculated polarization for different interlayer shifts. 

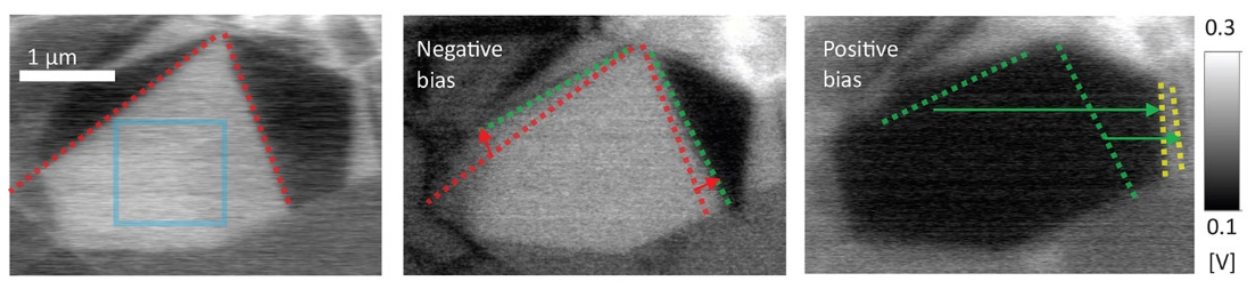

Fig. 3. Dynamic flipping of polarization orientation by domain-wall sliding. Kelvinprobe maps measured consecutively from left to right above a particular flake location showing domains of up (white) and down (black) polarizations. The middle image was taken after biasing the tip by a fixed DC voltage of -20 Volts and scanning it above the blue square region shown on the left-hand image. Then the tip was biased by 10 Volt and scanned again over the same region before taking the right-hand image. Consecutive domain-walls positions are marked by dashed red, green, and yellow lines. Larger white (black) domains appear after positive (negative) bias scans as a result of domain-wall motion beyond the scan area. Note that the number of domain-walls is apparently not altered. 